\section{WHAT IS THE COMMUNITY PHARMACIST'S ROLE IN PALLIATIVE CARE: VIEWS OF HEALTHCARE PROFESSIONALS WITHIN A UK CITY}

Elizabeth Miller, Julie D Morgan, Christina Wong, Alison Blenkinsopp. Sheffield Teaching Hospitals NHS Foundation Trust, University of Bradford

\subsection{6/bmjspcare-2018-ASPabstracts.9}

Background Changes in population demographics and greater demand for palliative care are placing increased pressure on general practitioners (GPs) to support patients and families near the end of life. There is little research on how community pharmacists (CPs) contribute and collaborate in the care of palliative care patients in the primary healthcare team. The aim of this study was to determine views of healthcare professionals on the CPs' role in palliative care.

Methods In-depth qualitative semi-structured interviews with a purposive sample of 16 community healthcare professionals. Participants were recruited through e-bulletins, email, team leaders and hospice team within Sheffield. Interviews were audio-recorded, transcribed verbatim and analysed using the Framework method following an inductive and iterative thematic process. Ethical approval was obtained from the University of Bradford.

Results 5 CPs, 3 GPs, 5 community nurses, 2 palliative care team members, and 1 intermediate care team member consented to interview. Two main themes emerged: timely access to palliative medicines and the CP's role in palliative care. There was poor understanding of the CP's professional role and pharmacy services of potential benefit to palliative care patients with nurses and GPs holding a traditional view of CPs in the dispensing and safe supply of medication. The CP's role was limited due to reluctance from other healthcare professionals to share information, poor access to patient records and a lack of integration into the primary healthcare team.

Conclusion Despite the national direction to increase the CPs involvement in palliative care, they remain a largely untapped resource for supporting patients and families towards the end of life due to a lack of integration into primary healthcare teams.

Acknowledgements This research was funded by Pharmacy Research UK (Grant reference GA10). The views expressed are those of the authors and not necessarily those of Pharmacy Research UK.

\section{Free papers 10-12 | Developing approaches to palliative care}

\section{BARRIERS TO PALLIATIVE CARE IN RESOURCE-LIMITED SETTINGS: A QUALITATIVE STUDY IN KAZAKHSTAN}

Kaisar Dauyey, Lyazzat Toleubekova, Byron Crape. Nazarbayev University School of Medicine

\subsection{6/bmjspcare-2018-ASPabstracts. 10}

Background Palliative care (PC) improves the quality of life of patients and their families facing life-threatening illness through relief of physical, emotional, and psychological suffering and pain. Identification of modifiable determinants of obstacles to universal quality PC in resource-poor settings like
Kazakhstan can represent an example for other countries with similar challenges in PC provision. The 2015 Quality of Death Index, which evaluates global distribution of availability and effectiveness of PC, places Kazakhstan 50th out of 80 countries assessed. The Association of Palliative Care of Kazakhstan reports a substantial unmet demand for increased accessibility to pain relief medications, increased numbers of PC facilities, and training courses for medical care professionals. These improvements are supported by legislation and national policies.

Specific Aims to identify specific determinants of obstacles to provision of PC in a resource-limited developing country including drug availability, opportunities for healthcare professionals to receive training in palliative medicine and policy development process, as well as to evaluate the status and cost-effectiveness of PC provision in Kazakhstan.

Methods The study employs qualitative research in form of purposive recruiting with content analysis, semi-structured indepth interviews until targeted participant numbers are completed or saturation is achieved. Consent was provided by participants. The data is then translated into English, coded and analysed. With the new study findings, supplemented by published literature and government documents, the best practices within the socio-cultural context of Kazakhstan are systematically determined.

Preliminary results The determinants of barriers to palliative care provision in Kazakhstan are associated with the limited availability of opioids, lack of specialist training and insufficient public awareness concerning palliation.

\section{DO WE PREPARE PATIENTS FOR THEIR DIGITAL LEGACY? A SURVEY OF HEALTHCARE PROFESSIONALS IN THE WEST MIDLANDS}

Hazel Coop, Clare Marlow. WM Cares, The Royal Wolverhampton NHS Trust

\subsection{6/bmjspcare-2018-ASPabstracts. 11}

Background Social media is becoming a more prominent part of our everyday lives. As a consequence we will be leaving a digital legacy after death. Digital legacy is the digital information that is available about someone following their death, which may include website or blog listings, social media profiles, photos, videos and gaming profiles. Many will find it a comfort that their legacy remains on social media but there can also be negative consequences in relation to death, dying and bereavement. This survey was carried out to establish whether we prepare patients for their digital legacy within the West Midlands.

Methods An online survey was sent to 11 hospices within the West Midlands over a 6 week period with an initial email and then a reminder at 3 weeks. The survey was circulated by the hospices to the doctors, nurses and social workers.

Results 210 responses were collected (35\% response rate). The majority of respondents were from nurses (77\%). Doctors accounted for $22 \%$ of the responses and $7 \%$ were from social workers. 96\% had never discussed digital legacy with patients. Reasons for not having such discussions largely related to it not being considered, lack of knowledge about digital legacy or it not being felt to be appropriate or important.

Discussion This survey of health and social care professionals shows that we do not discuss digital legacy with patients. There is a potential unmet need that we are not addressing 